\title{
Conserved Quantities of the One-Dimensional Hubbard Model
}

\author{
Eugenio Olmedilla ${ }^{(a)}$ and Miki Wadati \\ Institute of Physics, College of Arts and Sciences, University of Tokyo, Meguro-Ku, Tokyo 153, Japan \\ (Received 16 November 1987)
}

\begin{abstract}
We explicitly provide, in a simple way, the form of the conserved quantities of the one-dimensional Hubbard model. The method developed is based in a newly found factorization property of the local transition matrices, which is satisfied by several spin and fermion chain models. In this way, we have shown a systematic method for deriving the conserved quantities of integrable quantum systems.
\end{abstract}

PACS numbers: $05.30 .-\mathrm{d}, 05.50 .+\mathrm{q}, 75.10 . \mathrm{Lp}$

For several decades the Hubbard model has attracted the attention of physicists working in both solid-state of the model, found using the Bethe Ansatz, were studied by Lieb and Wu. ${ }^{1}$ However, the clarification of the algebraic structure underlying the model was initiated only recently. ${ }^{2,3}$

The tool for this algebraization is the quantum inverse scattering method, ${ }^{4}$ a method that places the theory of completely integrable quantum systems and of solvable statistical mechanical models in a unified framework. To a quantum integrable system we can associate the local transition matrix $\mathcal{L}_{n}(\lambda)$, which is part of a Lax pair of operators, such that

$$
\begin{aligned}
& \mathscr{R}(\lambda, \mu)\left[\mathcal{L}_{n}(\lambda) \otimes \mathcal{L}_{n}(\mu)\right] \\
&= {\left[\mathcal{L}_{n}(\mu) \otimes \mathcal{L}_{n}(\lambda)\right] \mathcal{R}(\lambda, \mu) ; }
\end{aligned}
$$

$\lambda, \mu$ are spectral parameters, and $\mathcal{R}(\lambda, \mu)$ is a $c$-number matrix. The Yang-Baxter relation (1) is a sufficient condition for the system to be integrable. The monodromy physics and mathematical physics. The eigenfunctions

matrix, for a chain with length $N$, is defined by

$$
\begin{aligned}
\mathcal{T}_{N}(\lambda) & =\mathcal{L}_{N}(\lambda) \mathcal{L}_{N-1}(\lambda) \cdots \mathcal{L}_{1}(\lambda) \\
& =: \prod_{n=1}^{N} \mathcal{L}_{n}(\lambda) .
\end{aligned}
$$

The relation (1) is also satisfied by $\mathcal{T}_{N}$ instead of $\mathcal{L}_{n}$. By use of an appropriate definition of trace in (1) with $\mathcal{T}_{N}$, it is easy to see that there exists a family of commuting matrices, denoted as transfer matrices,

$$
\left[T_{N}(\lambda), T_{N}(\mu)\right]=0,
$$

where $T_{N}$ is a trace of $\mathcal{T}_{N}$. The $\lambda$ expansion of $T_{N}(\lambda)$ gives a set of conserved operators $\left\{C_{j}\right\}$ which are involutive:

$$
\left[C_{i}, C_{j}\right]=0 .
$$

This is commonly understood as the condition for the complete integrability of a model.

The Hamiltonian of the one-dimensional Hubbard model is

$$
H=-\sum_{m, s}\left(a_{m+1, s}^{\dagger} a_{m, s}-a_{m+1, s} a_{m, s}^{\dagger}\right)+\frac{U}{4} \sum_{m}\left(2 n_{m \dagger}-1\right)\left(2 n_{m \downarrow}-1\right),
$$

where $s=\uparrow$ and $\downarrow$. The corresponding local transition matrix is

$$
\mathcal{L}_{m}(\theta)=\mathcal{L}(\theta)\left[\mathcal{L}_{m \dagger}(\theta) \underset{s}{\otimes} \mathcal{L}_{m \downarrow}(\theta)\right] \mathcal{L}(\theta),
$$

with

$$
\begin{aligned}
& \mathcal{L}(\theta)=\cosh \left[\frac{1}{2} h(\theta)\right] 1+\sinh \left[\frac{1}{2} h(\theta)\right] \sigma^{z} \otimes \sigma^{z}, \\
& \mathcal{L}_{m \uparrow}(\theta)=\left(\begin{array}{cc}
-i \alpha(\theta) n_{m \uparrow}-\gamma(\theta) a_{m \uparrow} a_{m \uparrow}^{\dagger} & i a_{m \uparrow} \\
a_{m \uparrow}^{\dagger} & -i \gamma(\theta) n_{m \uparrow}+\alpha(\theta) a_{m \uparrow} a_{m \uparrow}^{\dagger}
\end{array}\right), \\
& \mathcal{L}_{m \downarrow}(\theta)=\left(\begin{array}{cc}
i \alpha(\theta) n_{m \downarrow}+\gamma(\theta) a_{m !} a_{m \downarrow}^{\dagger} & a_{m \downarrow} \\
i a_{m \downarrow}^{\dagger} & i \gamma(\theta) n_{m \downarrow}-\alpha(\theta) a_{m \downarrow} a_{m \downarrow}^{\dagger}
\end{array}\right) .
\end{aligned}
$$

Here, 1 is the $4 \times 4$ identity matrix and as we are dealing with fermion operators, the direct product in (6) is a Grassmann product defined by

$$
[A \underset{s}{\otimes} B]_{\alpha \gamma, \beta \delta}=(-1)^{[P(\alpha)+P(\beta)] P(\gamma)} A_{\alpha \beta} B_{\gamma \delta},
$$


with $P(1)=0$ and $P(2)=1$. The coupling constant in the model (5), $U$, and the parametrization in (7) are related by

$$
4 \sinh [2 h(\theta)]=U \sin (2 \theta) .
$$

We also have

$$
\alpha(\theta)=\gamma(\theta+\pi / 2)=\sin (\theta+\pi / 2) .
$$

At $\theta=0$ the matrix operators $\mathcal{L}_{m, s}$ are

$$
\begin{aligned}
& \mathcal{L}_{m \uparrow}(0)=\left(\begin{array}{cc}
-i n_{m \uparrow} & i a_{m \uparrow} \\
a_{m \uparrow}^{\dagger} & a_{m \uparrow} a_{m \uparrow}^{\dagger}
\end{array}\right), \\
& \mathcal{L}_{m \downarrow}(0)-\left(\begin{array}{cc}
i n_{m \downarrow} & a_{m \downarrow} \\
i a_{m \downarrow}^{\dagger} & -a_{m \downarrow} a_{m \downarrow}^{\dagger}
\end{array}\right),
\end{aligned}
$$

and their $j$ th-order derivatives at $\theta=0$ are

$$
\mathcal{L}_{m, s}^{(j)}=\operatorname{sgn}(s)\left(\begin{array}{cc}
-\gamma_{j} a_{m, s} a_{m, s}^{\dagger}-i \alpha_{j} n_{m, s} & 0 \\
& \alpha_{j} a_{m, s} a_{m, s}^{\dagger}-i \gamma_{j} n_{m, s}
\end{array}\right),
$$

where $\alpha_{j}$ and $\gamma_{j}$ are the $j$ th-order derivatives of $\alpha(\theta)$ and $\gamma(\theta)$ at $\theta=0$, respectively, and $\operatorname{sgn}(s)=+1$ for $s=\uparrow$ and -1 for $s=\downarrow$.

In terms of the local transition matrix $\mathcal{L}_{m}(\theta)$, the monodromy matrix is

$$
\mathcal{T}(\theta)=\prod_{M=-p}^{r^{-}} \mathcal{L}_{m}(\theta)
$$

and the transfer matrix is defined by

$$
T(\theta)=\operatorname{str}[\mathcal{T}(\theta)]=\operatorname{tr}\left[\left(\sigma^{z} \otimes \sigma^{z}\right) \mathcal{T}(\theta)\right],
$$

where str denotes the supertrace of a matrix.

We define the operators

$$
\tilde{H}_{s}(\theta)=\sum_{m=-p}^{r} \tilde{H}_{m, m-1, s}(\theta),
$$

where $p$ and $r$ are the extremes of a chain of length $N=r+p+1$, with periodic boundary conditions, and

$$
\begin{aligned}
\tilde{H}_{m+1, m, s}(\theta)=1-n_{m+1, s} n_{m, s}-a_{m+1, s} a_{m+1, s}^{\dagger} a_{m, s} a_{m, s}^{\dagger}+\gamma(\theta)\left(a_{m+1, s} a_{m, s}^{\dagger}-a_{m+1, s}^{\dagger} a_{m, s}\right) & \\
& +\alpha(\theta)\left(n_{m+1, s} n_{m, s}+a_{m+1, s} a_{m+1, s}^{\dagger} a_{m, s} a_{m, s}^{\dagger}\right) .
\end{aligned}
$$

Expansion of $\tilde{H}_{m+1, m, s}(\theta)$,

$$
\tilde{H}_{m+1, m, s}(\theta)=\sum_{j=0}^{\infty} \tilde{H}_{m+1, m, s}^{(j)} \frac{\theta^{j}}{j !},
$$

gives

$$
\tilde{H}_{m+1, m, s}^{(0)}=1,
$$

and for $j \geq 1$

$$
\tilde{H}_{m+1, m, s}^{(j)}=\gamma_{j}\left(a_{m+1, s} a_{m, s}^{+}-a_{m+1, s}^{\dagger} a_{m, s}\right)+\alpha_{j}\left(n_{m+1, s} n_{m, s}+a_{m+1, s} a_{m+1, s}^{\dagger} a_{m, s} a_{m, s}^{\dagger}\right) .
$$

We further denote

$$
h_{m}=\left(2 n_{m \uparrow}-1\right)\left(2 n_{m \downarrow}-1\right) \text {. }
$$

With these notations, the Hubbard Hamiltonian (5) is

$$
H=\sum_{m, s} \tilde{H}_{m+1, m, s}^{(1)}+\frac{1}{4} U \sum_{m} h_{m} .
$$

With these definitions it is easy to prove the following relations between the local transition matrices $\mathcal{L}_{m}(\theta)$ and the $\tilde{H}_{s}(\theta)$ :

$$
\mathcal{L}_{m+1, s}(\theta) \mathcal{L}_{m, s}(0)=\mathcal{L}_{m+1, s}(0) \mathcal{L}_{m, s}(0) \tilde{H}_{m+1, m, s}(\theta), \quad \mathcal{L}_{m+1, s}(0) \mathcal{L}_{m, s}(\theta)=\tilde{H}_{m+1, m, s}(\theta) \mathcal{L}_{m+1, s}(0) \mathcal{L}_{m, s}(0) ;
$$

we also have for $j \geq 0$

$$
\sigma^{z} \mathcal{L}_{m, s}^{(j)}(0)=(-1)^{j} \mathcal{L}_{m, s}^{(j)}(0)\left(2 n_{m, s}-1\right), \quad \mathcal{L}_{m, s}^{(j)}(0) \sigma^{z}=(-1)^{j}\left(2 n_{m, s}-1\right) \mathcal{L}_{m, s}^{(j)}(0),
$$


and

$$
\left(2 n_{m+1, s}-1\right) \tilde{H}_{m+1, m, s}^{(j)}=(-1)^{j} \tilde{H}_{m+1, m, s}^{(j)}\left(2 n_{m+1, s}-1\right) .
$$

For a chain with periodic boundary conditions, definition (14) after taking account of (6) and (13) gives us

$$
T(\theta)=\sum_{j=0}^{N} \cosh ^{N-j} h(\theta) \sinh ^{j} h(\theta) \sum_{k_{1}=-p-1+j}^{r} \sum_{k_{2}}^{r-1} \ldots \sum_{-p-2+j}^{r-j+1} T_{k_{j}=-p}^{\mathbf{k}}(\theta) T_{l}^{\mathbf{k}}(\theta) .
$$

Here $\mathbf{k}=\left\{k_{1}, k_{2}, \ldots, k_{j}\right\}, k_{1}>k_{2}>\cdots>k_{j}$, and

$$
T_{s}^{\mathbf{k}}(\theta)=\operatorname{str}\left[\mathcal{L}_{r, s}(\theta) \cdots \sigma^{z} \mathcal{L}_{k_{1}, s}(\theta) \cdots \sigma^{z} \mathcal{L}_{k_{2}, s}(\theta) \cdots \sigma^{z} \mathcal{L}_{k, s}(\theta) \cdots \mathcal{L}_{-p, s}(\theta)\right] \text {, }
$$

and in particular

$$
T_{s}(\theta)=\operatorname{str}\left[\prod_{n=-p}^{r-} \mathcal{L}_{n, s}(\theta)\right] .
$$

It is convenient to introduce the following quantity:

$$
\hat{H}_{s}^{(l) \mathbf{k}}=\tilde{H}_{r, r-1, s}^{(l)}+\cdots+(-1)^{l} \tilde{H}_{k_{1}, k_{1}-1, s}^{(l)}+(-1)^{l} \tilde{H}_{k_{2}, k_{2}-1, s}^{(l)}+\cdots+(-1)^{l} \tilde{H}_{k_{,}, k,-1, s}^{(l)}+\cdots+\tilde{H}_{-p, r, s}^{(l)} .
$$

We observe that

$$
\hat{H}_{s}^{(2 l) \mathbf{k}}=\tilde{H}_{s}^{(2 l)} .
$$

After making use of (11), (23), and (24), we obtain

$$
T_{s}^{\mathbf{k}}(\theta) \simeq T_{s}(0)\left[1+\sum_{n=1}^{N-1} C_{n, s}^{\mathbf{k}} \theta^{n}\right] \prod_{m=k}^{j}\left(2 n_{k_{m}}-1\right) .
$$

The symbol $\simeq$ means equality up to order $N-1$ in $\theta$, and

$$
\begin{aligned}
& C_{1, s}^{\mathbf{k}}=\hat{H}_{s}^{(1) \mathbf{k}}, \quad C_{2, s}^{\mathbf{k}}=\hat{H}_{s}^{(2) \mathbf{k}} / 2 !+\hat{H}_{s}^{(1) \mathbf{k}} \circ \hat{H}_{s}^{(1) \mathbf{k}}, \\
& C_{3, s}^{\mathbf{k}}=\frac{\hat{H}_{s}^{(3) \mathbf{k}}}{3 !}+\frac{\hat{H}_{s}^{(2) \mathbf{k}}}{2 !} \circ \hat{H}_{s}^{(1) \mathbf{k}}+\hat{H}_{s}^{(1) \mathbf{k}} \circ \frac{\hat{H}_{s}^{(2) \mathbf{k}}}{2 !}+\hat{H}_{s}^{(1) \mathbf{k}} \circ \hat{H}_{s}^{(1) \mathbf{k}} \circ \hat{H}_{s}^{(1) \mathbf{k}}, \\
& C_{4, s}^{\mathbf{k}}=\frac{\hat{H}_{s}^{(4) \mathbf{k}}}{4 !}+\frac{\hat{H}_{s}^{(3) \mathbf{k}}}{3 !} \circ \hat{H}_{s}^{(1) \mathbf{k}}+\hat{H}_{s}^{(1) \mathbf{k}} \circ \frac{\hat{H}_{s}^{(3) \mathbf{k}}}{3 !}+\frac{\hat{H}_{s}^{(2) \mathbf{k}}}{2 !} \circ \frac{\hat{H}_{s}^{(2) \mathbf{k}}}{2 !}+\frac{\hat{H}_{s}^{(2) \mathbf{k}}}{2 !} \circ \hat{H}_{s}^{(1) \mathbf{k}} \circ \hat{H}_{s}^{(1) \mathbf{k}}+\hat{H}_{s}^{(1) \mathbf{k}} \circ \frac{\hat{H}_{s}^{(2) \mathbf{k}}}{2 !} \circ \hat{H}_{s}^{(1) \mathbf{k}} \\
& +\hat{H}_{s}^{(1) \mathbf{k}} \circ \hat{H}_{s}^{(1) \mathbf{k}} \circ \frac{\hat{H}_{s}^{(2) \mathbf{k}}}{2 !}+\hat{H}_{s}^{(1) \mathbf{k}} \circ \hat{H}_{s}^{(1) \mathbf{k}} \circ \hat{H}_{s}^{(1) \mathbf{k}} \circ \hat{H}_{s}^{(1) \mathbf{k}},
\end{aligned}
$$

where $\circ$ denotes the products of $\hat{H}_{s}^{(j) \mathbf{k}} / j$ ! in such a way that if two or more suffixes of the $\tilde{H}$ 's are connected [like $(j+1, j)$ and $(j, j-1)]$ their ordering cannot be broken. For instance, we have

$$
\tilde{H}_{6,5, s}^{\left(j_{1}\right)} \circ \tilde{H}_{4,3, s}^{\left(j_{2}\right)} \circ \tilde{H}_{2,1, s}^{\left(j_{3}\right)} \circ \tilde{H}_{1,6, s}^{\left(j_{4}\right)}=\tilde{H}_{2,1, s}^{\left(j_{3}\right)} \tilde{H}_{1,6, s}^{\left(j_{4}\right)} \tilde{H}_{6,5, s}^{\left(j_{1}\right)} \tilde{H}_{4,3, s}^{\left(j_{2}\right)}=\tilde{H}_{4,3, s}^{\left(j_{2}\right)} \tilde{H}_{2,1, s}^{\left(j_{3}\right)} \tilde{H}_{1,6, s}^{\left(j_{4}\right)} \tilde{H}_{6,5, s}^{\left(j_{1}\right)}
$$

We call such a product the connected product and $\circ$ is called the circle product. In general, $C_{n, s}^{\mathbf{k}}$ is the sum of the circle products of $\tilde{H}_{s}^{(j) \mathbf{k}} / j$ ! among themselves whose exponent $\operatorname{sum} \sum j$ is $n$.

In particular:

$$
C_{1, s}=\tilde{H}_{s}^{(1)}, \quad C_{2, s}=\tilde{H}_{s}^{(2)} / 2 !+\tilde{H}_{s}^{(1)} \circ \tilde{H}_{s}^{(1)}, \quad C_{3, s}=\tilde{H}_{s}^{(3)} / 3 !+\tilde{H}_{s}^{(2)} / 2 ! \circ \tilde{H}_{s}^{(1)}+\tilde{H}_{s}^{(1)} \circ \tilde{H}_{s}^{(2)} / 2 !+\tilde{H}_{s}^{(1)} \circ \tilde{H}_{s}^{(1)} \circ \tilde{H}_{s}^{(1)} .
$$

Finally, we arrive at

$$
\begin{aligned}
T(\theta) & =T(0) \sum_{j=0}^{N} \cosh ^{w-j} h(\theta) \sinh ^{j} h(\theta) \sum_{k_{1}=-p-1+j k_{2}=-p-2+j}^{r} \cdots \sum_{k_{j}=-p}^{r-j+1}\left[1+\sum_{m=1}^{N-1} C_{m \uparrow}^{\mathbf{k}} \theta^{m}\right]\left[1+\sum_{q=1}^{N-1} C_{q, \downarrow}^{\mathbf{k}} \theta^{q}\right] \prod_{t=1}^{j} h_{k_{t}} \\
& \simeq T(0)\left[1+\sum_{n=1}^{N-1} e_{n} \theta^{n}\right] .
\end{aligned}
$$

It is straightforward to write down the explicit forms of the conserved quantities $\mathcal{C}_{n}$. The first three conserved quan- 
tities are

$$
\begin{aligned}
& e_{1}=H, \quad e_{2}=C_{2 \uparrow}+C_{2 \downarrow}+C_{1 \uparrow} C_{1 \downarrow}+\frac{U}{4} \sum_{k=-p}^{r}\left(C_{1 \uparrow}^{k}+C_{1 \downarrow}^{k}\right) h_{k}+(N+2) \frac{U^{2}}{32}, \\
& e_{3=}=C_{3 \uparrow}+C_{3 \downarrow}+C_{2 \uparrow} C_{1 \downarrow}+C_{1 \uparrow} C_{2 \downarrow}+\frac{U}{4} \sum_{k=-p}^{r}\left(C_{2 \uparrow}^{k}+C_{2 \downarrow}^{k}+C_{1 \uparrow}^{k} C_{1 \downarrow}^{k}\right) h_{k}+\frac{U^{2}}{16} \sum_{k_{1}}^{r} \sum_{-p+1 k_{2}=-p}^{r-1}\left(C_{1 \uparrow}^{k_{1} k_{2}}+C_{1 \downarrow}^{k_{1} k_{2}}\right) h_{k_{4}} h_{k_{2}} \\
& +N \frac{U^{2}}{32}\left(C_{1 \uparrow}+C_{1 \downarrow}\right)+\left(N \frac{U^{2}}{64}-\frac{1}{3}\right) \frac{U}{2} .
\end{aligned}
$$

Formula (33) is also true with $T(0)$ to the right instead of the left.

For the infinite chain, we have expressions similar to (25), (30), and (33) for the monodromy matrix in place of the transfer matrix. Here we only mention that both trace and supertrace of the monodromy matrix give us an infinite set of conserved quantities. The properties that assure these results are

$$
\begin{aligned}
& \operatorname{tr} A=\operatorname{str}\left[\left(\sigma^{z} \otimes \sigma^{z} \otimes \sigma^{z} \otimes{ }^{z}\right) A\right], \\
& {\left[R\left(\theta, \theta^{\prime}\right), \sigma^{z} \otimes \sigma^{z} \otimes \sigma^{z} \otimes \sigma^{z}\right]=0,}
\end{aligned}
$$

where the matrix $\mathcal{R}\left(\theta, \theta^{\prime}\right)$ has been given in Ref. 3 .

In brief, we wish to remark on the simplicity of the purely algebraical method used to derive the conserved quantities (conserved operators). This method has proved to be systematic, and by using the quantum inverse scattering method $^{4}$ formulated on a lattice, we have been able to discuss spin models and fermion models on an equal footing. ${ }^{5}$

The key relations in our treatment are the commutation relations among the local transition matrices and the $\tilde{H}$ operators [see $(22)-(24)]$. We have obtained a compact formula for the transfer matrix which acts as the generator of the conserved quantities. Then, simply by expanding formula (25) in a power series of the spectral parameter, we can write down a sequence of conserved quantities.

Note that besides the $N-1$ conserved quantities that can be obtained by use of our method, the transfer matrix evaluated at $\theta=0, T(0)$, is also a conserved quantity.

Our conserved quantity $e_{2}$ (fermion model) and
Shastry's (boson model) second current $\left(H^{2}-i j\right.$ in Ref. 2) essentially coincide, up to a term that goes with $U^{2}$. We are unaware of a publication by Shastry which would allow us to discuss this in more detail.

We wish to thank Dr. Yasuhiro Akutsu and Dr. Kiyoshi Sogo for valuable discussions and critical reading of the manuscript. One of us (E.O.) is very grateful for the hospitality of the Institute of Physics, University of Tokyo, and for the financial support by the Japan Society for the Promotion of Science.

(a) Permanent address: Departamento de Métodos Matemáticos de la Física, Facultad de Ciencias Físicas, Universidad Complutense de Madrid, 28040 Madrid, Spain.

${ }^{1}$ E. Lieb and F. Y. Wu, Phys. Rev. Lett. 20, 1445 (1986).

${ }^{2}$ B. S. Shastry, Phys. Rev. Lett. 56, 1529, 2453 (1986).

${ }^{3}$ M. Wadati, E. Olmedilla, and Y. Akutsu, J. Phys. Soc. Jpn. 56, 1340 (1987); E. Olmedilla, M. Wadati, and Y. Akutsu, J. Phys. Soc. Jpn. 56, 2298 (1987).

${ }^{4}$ E. Olmedilla and M. Wadati, J. Phys. Soc. Jpn. (to be published).

${ }^{5}$ L. D. Faddeev, Sov. Sci. Rev. Math. Phys. C 1, 107 (1980); H. B. Thacker, Rev. Mod. Phys. 53, 253 (1981). P. P. Kulish and E. K. Sklyanin, in Integrable Quantum Field Theories, edited by J. Hietarinta and C. Montonen, Lecture Notes in Physics Vol. 151 (Springer-Verlag, Berlin, 1982), p. 61; L. A. Takhtadzhan and L. D. Faddeev, Russian Math. Surveys 34, 11 (1979); M. Wadati, in Dynamical Problems in Soliton Systems, edited by S. Takeno (Springer-Verlag, Berlin, 1983), p. 68; M. Wadati and Y. Akutsu, "Exactly Solvable Models in Statistical Mechanics," edited by M. Lakshmann, Lecture Notes in Physics (Springer-Verlag, Berlin, to be published). 